\title{
OIL SPILL DISPERSION IN FULL-POLARIMETRIC AND HYBRID-POLARITY SAR
}

\author{
Camilla Brekke, Stine Skrunes, and Martine M. Espeseth \\ UiT The Arctic University of Norway, Dept. of Physics and Technology, \\ PO box 6050 Langnes, 9037 Troms $\varnothing$, Norway
}

\begin{abstract}
We show that indications of spatial dispersion effects on mineral oil slicks are observed by space-borne multipolarization synthetic aperture radar. This is readily perceived by eye when correlating multipolarization synthetic aperture radar observables with the ship track of the dispersion vessel. We investigate real full-polarimetric (linear transmit/linear receive) as well as simulated and real hybrid-polarity (circular transmit/linear receive) synthetic aperture radar features. Specifically, we give a thorough discussion of observables derived from the cross-correlation of two polarization channels, and compare their counterparts in the two above mentioned polarization bases.
\end{abstract}

Index Terms - SAR, polarimetry, oil spills

\section{INTRODUCTION}

During large-scale oil spill recovery situations, it is important to establish an overview picture to aid the on-ground manmade recovery effort to target the parts of the slick characterized by higher volumetric oil fraction or greater thickness of oil. Oil spill dispersion at sea helps to break the oil up into small droplets that could speed up the biodegradation process. The novelty of this work is that we reveal a possible correlation between multipolarization synthetic aperture radar (SAR) features and the trail of ship dispersion of an experimental spill. The study is done on a pair of Radarsat-2 (RS-2) quad-polarimetric and RISAT-1 (RI-1) hybrid-polarity SAR scenes overlapping in time and space and covering oil spills released during Norwegian Clean Seas Association for Operating Companies' annual oil-on-water (OOW) exercise in the North Sea. We describe the first results from the campaign that took place in June 2016 at the Frigg field. We compare multipolarization SAR features extracted from i) real quad-polarimetric, ii) simulated hybrid-polarity, and iii) real hybrid-polarity radar measurements.

This work is partly funded through The Research Council of Norway (grant number 237906 and 233896).

\section{THEORY}

In a full-polarimetric SAR system, the acquisition strategy is based on a quasi-simultaneous transmission of two linear orthogonal polarized waves followed by reception in two linear orthogonal polarization channels with the same phase reference. We will refer to this architecture as linear on transmit and linear on receive (LTLR) polarization basis. For each pixel, and assuming reciprocity $(\mathrm{HV}=\mathrm{VH})$, the resulting information is stored in a scattering matrix:

$$
\mathbf{S}_{L T L R}=\left[\begin{array}{ll}
S_{H H} & S_{H V} \\
S_{H V} & S_{V V}
\end{array}\right],
$$

where $S$ is a complex scattering coefficient. The subscripts indicate the polarization on transmit and reception; $\mathrm{H}$ is linear horizontal polarization and $\mathrm{V}$ is linear vertical polarization. The $\mathbf{S}$ matrix can be represented as the lexicographic vector

$$
\bar{k}_{L T L R}=\left[S_{H H}, \sqrt{2} S_{H V}, S_{V V}\right]^{T},
$$

where $T$ represents the transpose operator.

The SAR systems studied in this work are either LTLR or circular on transmit and linear on receive (CTLR) polarizations, exemplified by data products from RS-2 quadpolarimetric mode and RI-1 hybrid-polarity mode, respectively. We also simulate CTLR data for direct comparison with the acquired LTLR data from RS-2. The transformation from LTLR to simulated CTLR is performed based on relating the CTLR scattering vector to the $\mathbf{S}_{L T L R}$ matrix (see, e.g., [1]) :

$$
\bar{k}_{C T L R}=\left[\begin{array}{c}
S_{R H} \\
S_{R V}
\end{array}\right]=\frac{1}{\sqrt{2}}\left[\begin{array}{l}
S_{H H}-i S_{H V} \\
S_{H V}-i S_{V V}
\end{array}\right],
$$

where $i$ is the imaginary unit and $\mathrm{R}$ represents right circular polarization.

The covariance matrices in LTLR and CTLR are obtained by computing the outer products of the scattering vectors:

$$
\mathbf{C}_{L T L R}=\left\langle\bar{k}_{L T L R} \cdot \bar{k}_{L T L R}^{* T}>\right.
$$

and

$$
\mathbf{C}_{C T L R}=\left\langle\bar{k}_{C T L R} \cdot \bar{k}_{C T L R}^{* T}\right\rangle,
$$


where complex conjugate is indicated by the $*$ symbol and $<\cdot>$ is averaging over a neighbourhood of $N$ pixels. The elements of the covariance matrices are derived from crosscorrelation terms on the form $S_{x} S_{y}^{*}$, where $\{x, y\}$ represents the system's combination of polarization at transmit and receive. We define the Hermitian product as [2]:

$$
\rho_{x, y}=S_{x} S_{y}^{*}=r_{x} r_{y} \exp \left(i\left(\phi_{x}-\phi_{y}\right)\right),
$$

where $z_{x, y}=r_{x} r_{y}$ is the amplitude, and $\phi_{x, y}=\phi_{x}-\phi_{y}$ is the phase difference. In the proceeding sections, we investigate observables derived from the cross-correlation of two polarization channels and its constituents. Both LTLR and CTLR polarization bases are discussed.

\subsection{Observables}

Working in the LTLR polarization basis, the absolute value of the real part of the copolarization ( $\mathrm{HH}$ and VV) cross-product was identified as a powerful feature for oil spill studies (see [3], and references therein). The observable is defined as:

$$
r_{\rho_{H H, V V}}=\left|\mathfrak{R}\left(<\rho_{H H, V V}>\right)\right|,
$$

where $|\cdot|$ represents the absolute value and $\mathfrak{R}$ the real part.

Moving to a CTLR polarization basis, we showed in [4] that the absolute value of the imaginary part of the crosscorrelation between $\mathrm{RH}$ and $\mathrm{RV}$ resemble the observable in eq. (7);

$$
i_{\rho_{R H, R V}}=\left|\Im\left(\left\langle\rho_{R H, R V}\right\rangle\right)\right|,
$$

where $\mathfrak{I}$ is the imaginary part.

In this work, we also pay attention to the standard deviation of the cross-correlation amplitude in the LTLR polarization basis (and comparable for CTLR, but not shown here):

$$
\sigma_{z_{H H, V V}}=\sqrt{\left\langle\left(z_{H H, V V}\right)^{2}\right\rangle-\left(<z_{H H, V V}>\right)^{2}} .
$$

In LTLR polarization basis (and comparable for CTLR, but not shown here), the standard deviation of the copolarization phase difference is defined as:

$$
\sigma_{\phi_{H H, V V}}=\sqrt{\left\langle\left(\phi_{H H, V V}\right)^{2}\right\rangle-\left(\left\langle\phi_{H H, V V}\right\rangle\right)^{2}}
$$

This observable predominantly reveals internal zones of the slick that are assumed to be thick enough to have an impact on the dielectric properties (image not shown here).

Numerous features can be derived from SAR. We confine our study to those listed in Tab. 1, as they cover complementary information about radiometry and coherency. Feature \#3 and \#6 are derived from ratios of the cross-correlation terms, i.e., the phase angle is the arctan of the imaginary divided by the real part. Hence, in the tilted Bragg model, $\mathrm{f} 3$ and $\mathrm{f} 6$ will be independent of the damping of gravity-capillary waves by the oil, while sensitive to the dielectric constant, the slope, and the incidence angle. Feature \#1-2 and \#4-5 are not composed of ratios, and are dependent on the damping of gravitycapillary waves by the oil, the dielectric constant, the slope, and the incidence angle (see, e.g, [5] for discussion).
Table 1. Multipolarization SAR features.

\begin{tabular}{lll|lcc}
\hline \hline Basis & $\mathrm{f} \#$ & feature & Basis & f\# & feature \\
\hline LTLR & $\mathrm{f} 1$ & $r_{\rho_{H H, V V}}$ & CTLR & f4 & $i_{\rho_{R H, R V}}$ \\
LTLR & f2 & $\sigma_{z_{H}, V V}$ & CTLR & f5 & $\sigma_{z_{R H, R V}}$ \\
LTLR & f3 & $\sigma_{\phi_{H H, V V}}$ & CTLR & f6 & $\sigma_{\phi_{R H, R V}}$ \\
\hline
\end{tabular}

\section{PAIRED SAR SCENES OF DISPERSED SLICKS}

In this study, we investigate a pair of RS-2 and RI-1 SAR scenes. The images within the pair are partly overlapping geographically with a small temporal difference between the times of acquisition (28 minutes). See Fig. 1 and some image
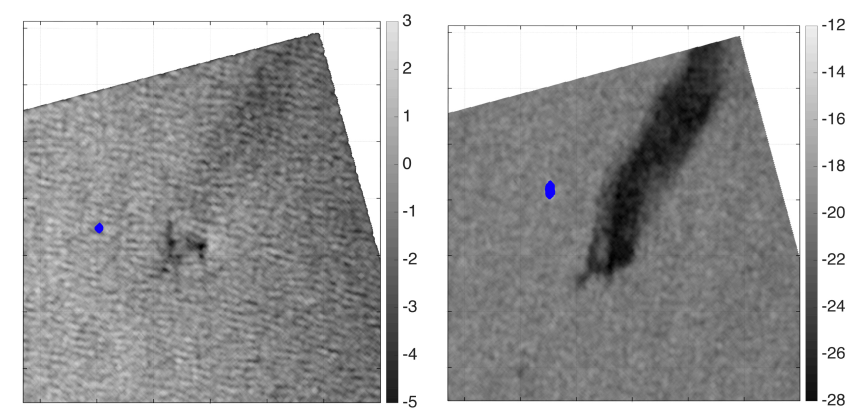

Fig. 1. Intensity images of RI-1 RV (left) and RS-2 VV. Only a piece of the overlap section between the RS-2 scene and the RI-1 scene is shown. A ship in near vicinity of the slick is masked out (blue dot). RISAT-1 2016-Antrix, processed by KSAT, all rights reserved 2016. RADARSAT-2 Data and Products MDA LTD. (2016) - All Rights Reserved.

details given in Table 2 .

Table 2. Paired SAR acquisitions from OOW2016. The incidence angle is denoted by $\theta$. FQ22: Fine resolution Quadpolarization beam 22. FRS-1: Fine Resolution Stripmap-1.

\begin{tabular}{llll}
\hline \hline Sensor \& mode & Date \& time & $\theta$ & Slick type \\
\hline RI-1 FRS-1 & 15 June 17:07 UTC & $14^{\circ}$ & Mineral \\
\hline RS-2 FQ22 & 15 June 17:35 UTC & $42^{\circ}$ & Mineral \\
\hline
\end{tabular}

On the 15 June, $8 \mathrm{~m}^{3}$ of Åsgard Blend (a North Sea crude oil type) was released at the site. This happened at 08:0508:10 UTC, several hours before the overpass of RI-1 at 17:07 UTC and RS-2 at 17:35 UTC. During release, the wind came from NNE at a wind speed of 6-7 m/s. In the hours before the satellite overpass, the wind speed increased to $11-14 \mathrm{~m} / \mathrm{s}$ from NNE. The wave height this day was $1.25-2.5 \mathrm{~m}$. The RS2 scene contains several slicks, including the Åsgard Blend slick and remains from releases the day before, while the RI1 scene only contains the Åsgard Blend slick.

After the oil was introduced, human interventions took place in the form of dispersions of the Asgard Blend slick. 


\subsection{Track of dispersing ship}

On June 15, M/S Strilborg dispersed the oil slick twice. The first dispersion took place in the time frame of 10:55 - 12:00 UTC, while the last treatment took place 16:40 - 16:50 UTC only minutes before the satellite SAR acquisitions. The ship's positions were recorded by the automatic information system (AIS) and are here overlaid the SAR VV polarization intensity image, as show in Fig. 2 (top panel).
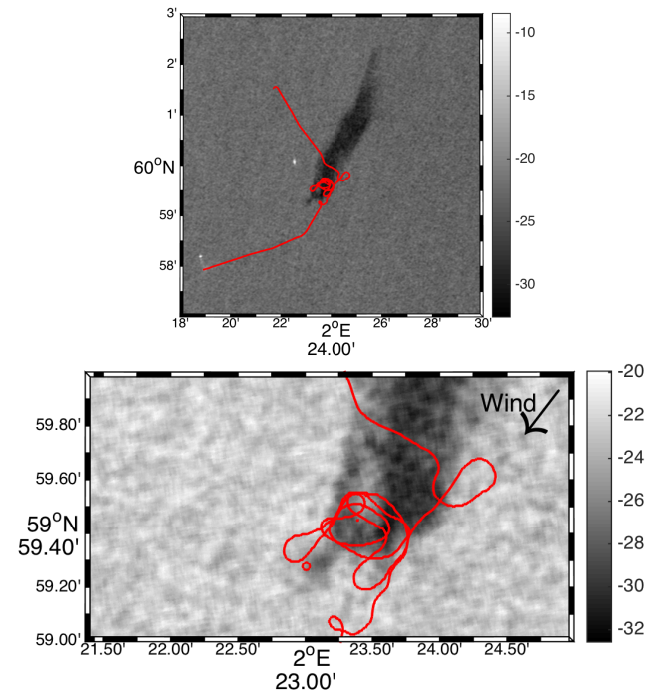

Fig. 2. Top: Track of M/S Strilborg overlaid the oil spill in the RS-2 intensity image (dB) at 17:35 UTC. The track starts at 16:00 UTC to the north-west and ends at 17:35 UTC to the south-west of the slick. Bottom: The corrected track is overlaid a zoom-in on the oil slick. Red dot: corrected dispersion position at 16:41. Red circle: corrected dispersion position at 16:48. Wind direction indicated.

Because of the evolving nature of the slick and its wind (and possibly to a lesser extent current [6]) driven transport on the ocean surface, the ship track has to be corrected to fit with the georeferenced SAR scene. This procedure is not trivial, but a best-effort attempt has been made by carefully investigating video clips from a Finnish surveillance aircraft monitoring the dispersion operation. We identified one approximate location of the ship within the slick prior to the SAR acquisition, by combining the latitude/longitude from the AIS logs, the latitude/longitude positions recorded by the aircraft, and the slick's visual appearance and shape from the aircraft video material. This approximate ship location was then used as a tie point to linearly shift the whole ship track accordingly (see bottom panel of Fig. 1).

As evident by a close inspection of the bottom panel in Fig. 2 (and figures later shown in section 4), the outcome turns out to be reasonable with respect to a) the spatial arrangement of the slick and b) the wind direction indicated in the right panel of Fig. 1. Note that the dispersion took place on the right side of the ship; hence, there is an offset between the ship track and the targeted area to the right.

The geographically corrected ship track is used next in the interpretation and discussion of the SAR observables.

\section{RESULTS AND DISCUSSIONS}

The RI-1 scene is acquired at very low incidence angles, and more specular reflections may occur, yielding a weak contrast between the majority of the slick and the ambient water (see Fig. 1). Even though the results are still interesting, for this reason, the RI-1 scene is left out of the following discussions.

For RS-2, four of the observables (f1, f2, f4, and f5) in Table 1 are visualized (on log scale) in Fig. 3. Different internal variations are visible in $\mathrm{f} 1$ vs. $\mathrm{f} 2$ and in $\mathrm{f} 4 \mathrm{vs.}$. f5. In particular, we see a distinct dark blue region in $\mathrm{f} 2$ and $\mathrm{f} 5$ with a sharp contrast to the lighter blue region. Also note the "hole", which is clearly visible in $\mathrm{fl}$ in the southern part of the slick, at approximate pixel position $(1000,250)$. This small internal region has values closer to the ambient sea surface as compared to the other slick pixels.

The four panels of Fig. 4 are zoomed-in on the recently dispersed part of the slick. The ship track is here overlaid the images for easier comparison. We can clearly see that there is some correlation between the ship track and the spatial zoning within the slick. We would like to highlight the area where the ship has traversed the slick. Here we can clearly see a pattern within the slick, e.g., in $\mathrm{f} 2$ and $\mathrm{f} 5$, matching the route of the dispersing ship.

\section{CONCLUSIONS}

To the authors' knowledge, there are few reports of signatures related to dispersants in radar imagery (see [7]). This work shows that dispersion effects on oil spills may be visible in multipolarization SAR imagery from space. Six multipolarization features are explored. The features are based on the cross-correlation of pairs of polarization channels; $\mathrm{HH}$ and VV in LTLR and RH and RV in CTLR. The standard deviation of the cross-correlation amplitude is proposed as a promising observable; capturing the internal variance of the radiometry within the slick. Based on visual interpretation, this feature performs comparable in the two polarization bases.

\section{ACKNOWLEDGEMENT}

The Radarsat-2 data was provided by NSC/KSAT, NorwegianCanadian RADARSAT agreement 2016. We thank NOFO for letting us participate in the exercise, and NOFO and SINTEF for providing in situ information. Thanks also to Air Patrol Squadron Finland/SYKE for aerial photo and video material utilized in this study, and to the Norwegian Coastal Administration for help with obtaining AIS information. 

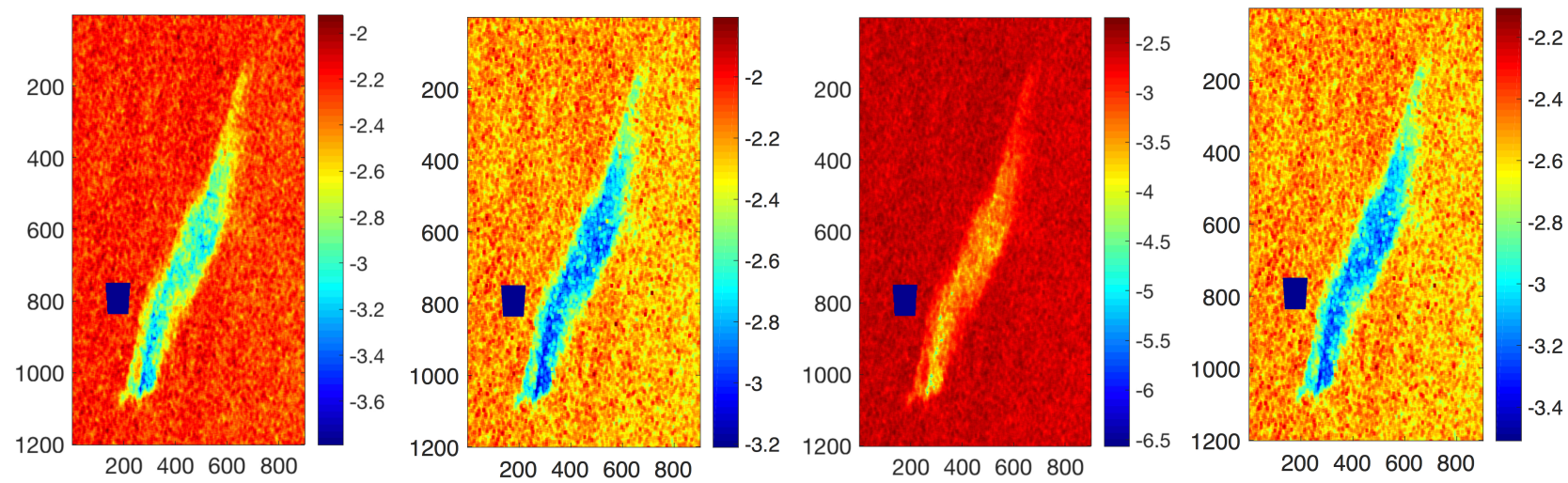

Fig. 3. The panels show f1, f2, f4, and f5 (from left to right). A ship in near vicinity of the slick is masked out (blue dot). The images are log transformed for better visual interpretation.

\section{REFERENCES}

[1] M. E. Nord, T. L. Ainsworth, J. S. Lee, and N. J. S. Stacy, "Comparison of compact polarimetric synthetic aperture radar modes," IEEE Trans. Geosci. Remote Sens., vol. 47, no. 1, pp. 174-188, Jan. 2009.

[2] R. J. A. Tough, D. Blacknell, and S. Quegan, "A statistical description of polarimetric and interferometric synthetic aperture radar data," Proc. R. Soc. Lond. A., vol. 449, no. 1937, pp. 567-589, June 1995.

[3] S. Skrunes, C. Brekke, and T. Eltoft, "Characterization of Marine Surface Slicks by Radarsat-2 Multipolarization Features," IEEE Trans. Geosci. Remote Sens., vol. 52, no. 9, pp. 5302 - 5319, Sept. 2014.

[4] C. Brekke, C. E. Jones, S. Skrunes, B. Holt, M. Espeseth, and T. Eltoft, "Cross-Correlation Between Polarization Channels in SAR Imagery Over Oceanographic Features," IEEE Geosci. Remote Sens. Lett., vol. 13, no. 7, pp. 997 - 1001, July 2016.

[5] M. M. Espeseth, S. Skrunes, C. E. Jones, C. Brekke, B. Holt, and A. P. Doulgeris, "Analysis of Evolving Oil Spills in Full-Polarimetric and Hybrid-Polarity SAR," IEEE Trans. Geosci. Remote Sens., 2017 (accepted).

[6] C. Jones, K.-F. Dagestad, Ø. Breivik, B. Holt, J. Röhrs, K. Christensen, M. Espeseth, C. Brekke, and S. Skrunes, "Measurement and modeling of oil slick transport," IEEE Trans. Geosci. Remote Sens., vol. 121, no. 10, pp. 7759 7775, Oct. 2016.

[7] C. E. Jones, B. Minchew, B. Holt, and S. Hensley, "Studies of the Deepwater Horizon oil spill with the uavsar radar," Monitoring and Modeling the Deepwater Horizon Oil Spill: A Record Breaking Enterprise. Geophysical Monograph. No.195. American Geophysical Union, Washington, DC, pp. 33-50, 2011.
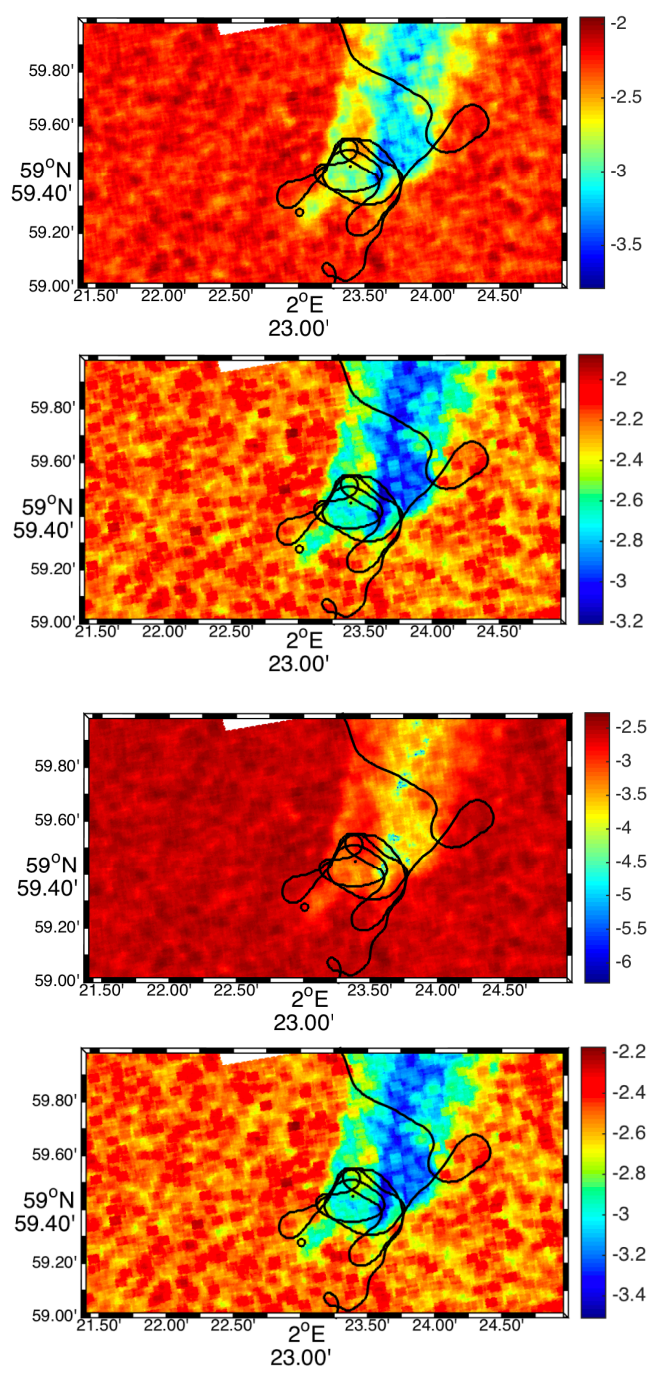

Fig. 4. Zoom in on f1-2 and f4-5 (from top to bottom). Track of ship overlaid. Black dot: corrected dispersion position at 16:41. Black circle: corrected dispersion position at 16:48. 